\title{
The Combined Use of Chemotherapy and Radiotherapy with PD-I Inhibitor, Pembrolizumab, in Advanced Cervical Cancer: A Case Report
}

This article was published in the following Dove Press journal: OncoTargets and Therapy

\author{
Mengmeng Lyu \\ Yang Shen \\ Nitish Beharee (D) \\ Jin Lu \\ Fei Deng \\ Jinhua Wang
}

Jiangsu Cancer Hospital and Jiangsu Institute of Cancer Research and the Affiliated Cancer Hospital of Nanjing Medical University, Department of Gynecologic Oncology, Nanjing, Jiangsu Province, People's Republic of China
Correspondence: Fei Deng; Jinhua Wang Email dengfeifine@sina.com; Wangjinhua588@163.com

\begin{abstract}
As a result of the limited therapeutic options, advanced cervical cancer is difficult to treat, making the prognosis poor. Therefore, new therapeutic modalities or combinations need to be explored. We herein reported a case of stage IVB cervical cancer which was irresponsive to chemotherapy alone. Based on previous studies and after patient's consent was obtained, we made a therapeutic plan: chemotherapy (albumin-bound paclitaxel and carboplatin) combined with immunotherapy (PD-1 inhibitor pembrolizumab). After 6 cycles of combined treatment, the patient got almost complete resolution with slight advent event. The treatment was further supported by local radiotherapy combined with immunotherapy. During the treatment period, disease was relatively stable, but the patient suffered severe grade 4 myelosuppression. We were therefore left with no other choice than to interrupt both chemotheraphy and radiotherapy. Before long, the tumor grew explosively again. These guided us to conclude that the combination use of albumin-bound paclitaxel (nabpaclitaxel) and carboplatin and pembrolizumab is effective and well tolerated in the treatment of advanced cervical cancer. The combined use of radiotherapy and pembrolizumab may also be effective. However, the combination use of chemotherapy, radiotherapy and immunotherapy in advanced cancer has not been well studied, and there are still many unsolved queries.
\end{abstract}

Keywords: cervical cancer, PD-1 inhibitor, pembrolizumab, chemotherapy, radiotherapy

\section{Introduction}

With the use of HPV (Human Papillomavirus) vaccines and cervical cancer screening, the incidence and the mortality rate of cervical cancer have been decreased in many countries worldwide. ${ }^{1,2}$ However, cervical cancer still ranks fourth for both incidence and mortality in female cancers and is the most common gynecological tumor, with an incidence of 569847 new cases and 311365 deaths reported in the year 2018 worldwide as estimated by GLOBOCAN. ${ }^{2}$ Patients with localized disease could select radical and surgical treatment, while the treatment options for patients with advanced disease including metastatic (ie FIGO stage IVB) and recurrent cervical cancer is limited, and the prognosis is poor. ${ }^{3,4}$ A common option for patients with advanced cervical cancer is palliative chemotherapy mainly based on cisplatin. The combination use of vascular endothelial growth factor inhibitors is also found to be functional. However, the response rate is not satisfactory. ${ }^{5-8}$ New treatment modalities are needed to be developed. With the development of immunotherapy, the effect of the Programmed cell death-1/programmed cell death-ligand 1 (PD-1/PD-L1) inhibitors, 
one of the most well-known immune-checkpoint inhibitors, has been researched in cervical cancer.

The PD-1/PD-L1 inhibitors have proven to be beneficial in various tumors including cervical cancer. ${ }^{9}$ Briefly, PD-1 expresses on the surface of T cells, while PD-L1 expresses itself on the surface of tumor cells. The binding of PD-1 and PD-L1 triggers immune tolerance to tumor cells and promotes tumor growth. PD-1/PD-L1 inhibitors re-energize the immune response by blocking PD-1/PD-L1 binding. ${ }^{10}$ The antitumor activity and manageable safety of PD-1/PD-L1 inhibitors including pembrolizumab and nivolumab was verified by Clinical trial KEYNOTE-028, KEYNOTE-158 and CheckMate 358 in advanced cervical cancer. ${ }^{11-13}$ And the FDA has approved pembrolizumab for patients with recurrent or metastatic cervical cancer with disease progression during or after chemotherapy. ${ }^{9}$ However, the response rate of monotherapy of PD-1/PD-L1 inhibitors for advanced cervical cancer was not high, the overall response rate was $17 \%$, $13.3 \%$ and $26.3 \%$ respectively for clinical trial KEYNOTE028, KEYNOTE-158 and CheckMate 358. ${ }^{11-13}$ Many ongoing clinical trials investigated the combination use of PD-1/PD-L1 inhibitors with chemotherapy including Platinum, Vinorelbine, radiotherapy and vascular endothelial growth factor inhibitors, in an aim to enhance the efficacy of PD-1/PD-L1 inhibitors treatment. ${ }^{9}$

Here, we reported a case of advanced cervical cancer of FIGO stage IVB. The patient reached almost complete remission after receiving combination treatment of chemotherapy (nab-paclitaxel and carboplatin) and immunotherapy (pembrolizumab). As the systemic metastasis was under control, the patient was transferred to local pelvic palliative radiotherapy combined with immunotherapy. The disease remained stable, however, severe adverse events occurred during this period and both chemotherapy and radiotherapy had to be interrupted. The tumor explosively grew again. We herewith want to share the successful combination use of nab-paclitaxel and carboplatin, and PDlinhibitor together, and discuss the clinical thinking brought by this case.

\section{Case Presentation}

A 55-year-old female patient with no past medical histories went to hospital with chief complaint of increasing amount of watery vaginal discharge for the past one month. Following cervical biopsy done in August 2018, the woman was diagnosed with cervical malignant tumor. Histopathological report showed that it was a poorly or moderately differentiated invasive squamous cell carcinoma with locally visible adenoid structure. The patient received one course of chemotherapy with cisplatin and paclitaxel. However, three weeks later, the cervical lesion was found to be larger, and the patient was transferred to our hospital. The treatment timeline is shown in Figure 1.

When the patient attended our hospital, gynecological examination showed that the anterior wall of the vagina was invaded up to the lower third. The cervical tumor was ulcerated, with a diameter of $4 \mathrm{~cm}$. The bilateral parametrium was infiltrated up to the pelvis. A comprehensive inspection including CT and PET-CT was performed in our hospital. Computed tomography (CT) scan (Figure 2A) of the chest and abdomen revealed an irregular cervical mass measuring $4.16^{*} 4.15 \mathrm{~cm}$, with invasion of the vagina, and infiltration of the bilateral parametrium up to the pelvis. Dense masses were seen in bilateral adnexa $\left(3.18^{*} 2.19 \mathrm{~cm}\right.$ in the right side and $2.62 * 2.77 \mathrm{~cm}$ in the left side) favoring the risk of metastasis. Multiple nodules were observed in the uterine rectal fossa indicating multiple implants. And the enhanced mass showed liver metastases (the largest one measuring $1.23 * 1.27 \mathrm{~cm})$. Multiple enlarged lymph nodes were observed in the retroperitoneal area, around the left iliac

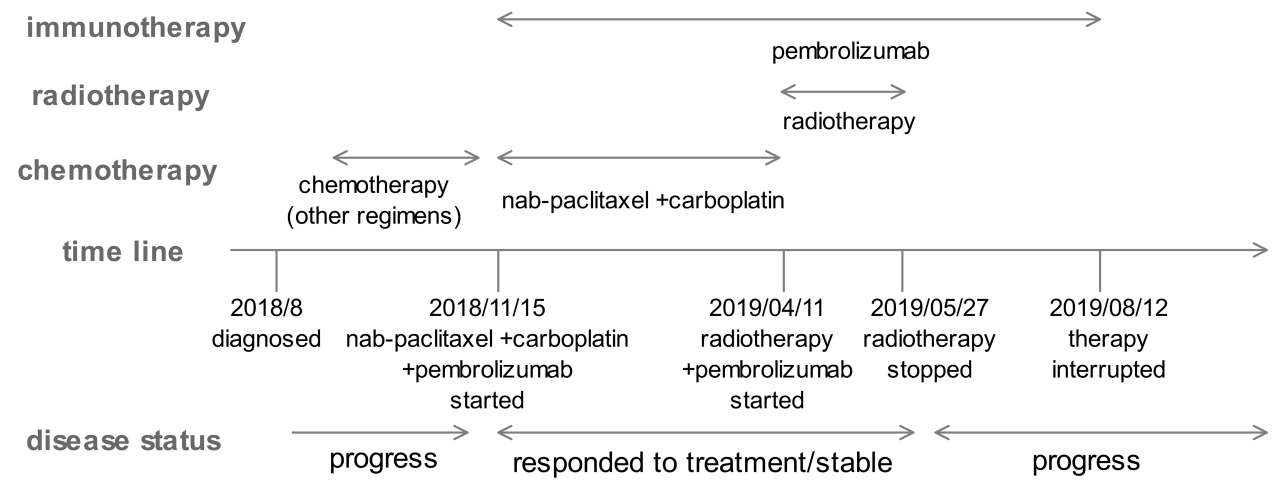

Figure I Timeline of different treatments and disease status. 


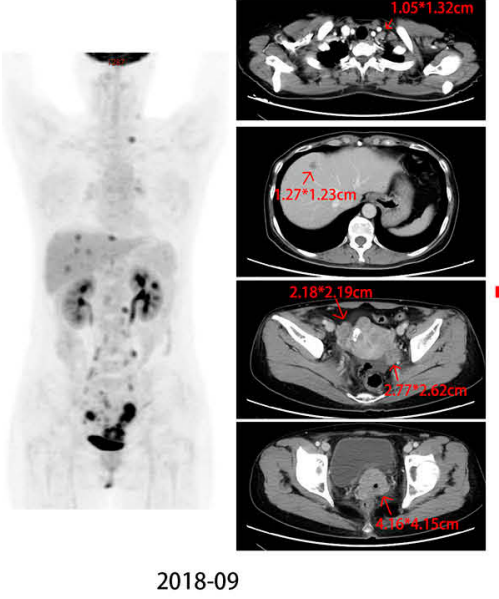

A

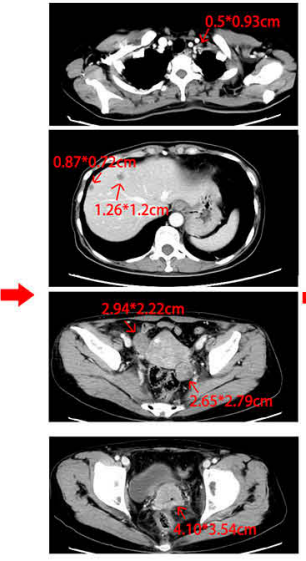

2018-11

B

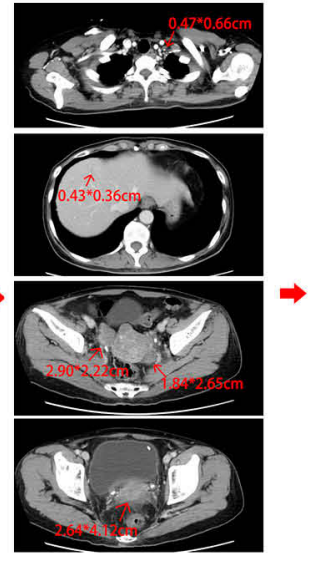

2019-01

C
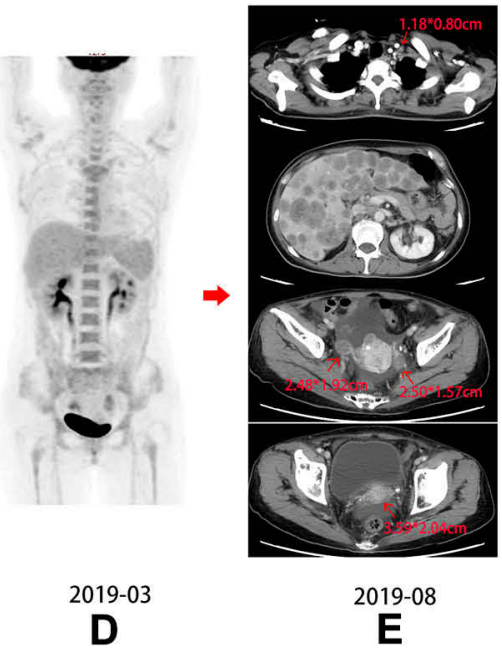

2019-08

E

Figure 2 PET-CT and CT images of the patient. (A) The patient transferred to our hospital, (B) after chemotherapy of other regimens, (C) after two cycles of combination treatment of nab-paclitaxel and carboplatin and pembrolizumab, (D) after 6 cycles of combination treatment of nab-paclitaxel and carboplatin and pembrolizumab, (E) explosive growth of the tumor.

vessels and pelvic wall. The left supraclavicular lymph nodes presented swelling measuring $1.32 * 1.05 \mathrm{~cm}$. The positron emission tomography-computed tomography (PET/CT) (Figure 2A) showed cervical cancer with invasion in lower uterus and vaginal invasion. The PET/CT also showed positive metastatic evidence in the left supraclavicular region, bilateral parametrium, liver, retroperitoneal lymph nodes, areas around the iliac vessels and pelvic wall. Tumor biomarker CA-125 and CEA were high, while SCC was normal (Figure 3). And the patient was diagnosed with cervical cancer IVB according to FIGO staging with a TNM classification established as T3bN1M1.

The clinical characteristics of the disease resembled that of small cell neuroendocrine cervical carcinoma (SCNECC), by having a systemic metastasis, by being a rapid progressing tumor, and by not being sensitive to chemotherapy regimen of cisplatin and Paclitaxel. ${ }^{14}$ The pathological type of SCNESS was suspected, and we empirically used the chemotherapy regimen of etoposide and cisplatin (EP) in September 2018. At the same time, we added the immunohistochemical examination of the cervical biopsy tissue and PD-L1 examination. PD-L1 status was positive, PD-L1 was presented in $10 \%$ of tumoral cells. And the blood tumor mutation burden was $12.0 \mathrm{Muts} / \mathrm{Mb}$. Immunohistochemical work-up revealed positive expression CK5/6-, P63 \pm , P40-, P16+, CK7++, WT-1 \pm , P53++90\%, Ki67+70\%. The histopathological report indicated poorly differentiated adenocarcinoma (Figure 4). After one session of the EP

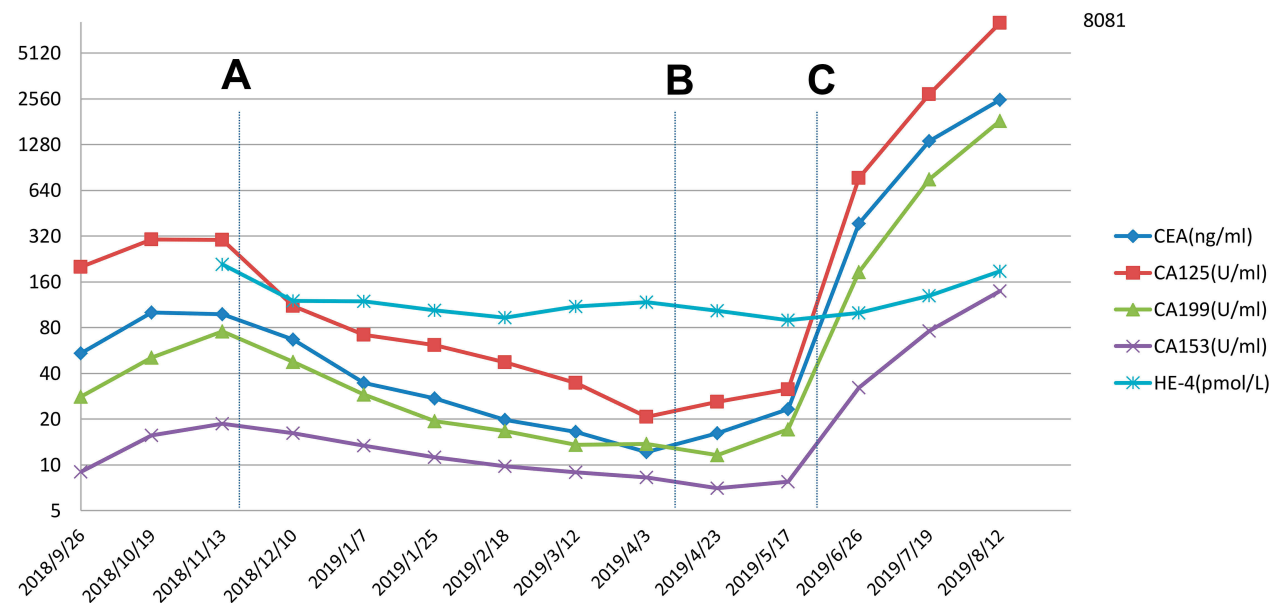

Figure 3 Changes in tumor index values. The ordinate is displayed on a logarithmic scale. (A) The patient started combination treatment of chemotherapy and immunotherapy. (B) The patient was transferred to radiotherapy combined with immunotherapy. (C) Radiotherapy was interrupted and the patient started single use of pembrolizumab. 


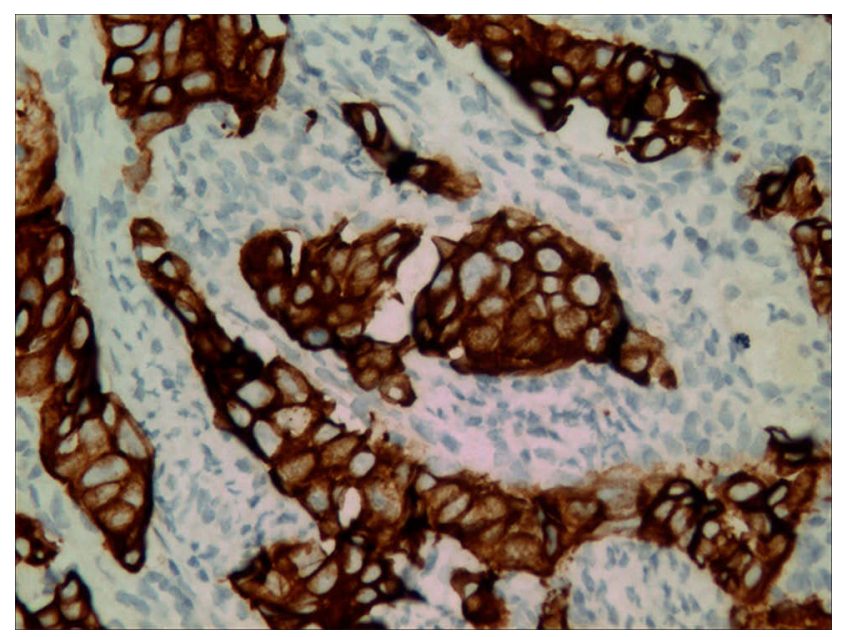

Figure 4 Representative picture of immunohistochemical staining. The picture revealed positive CK7 staining which showed brown color (magnification, $\times 400$ ).

chemotherapy, the cervical lesion upon gynecological examination was found to be slightly reduced, and bevacizumab was added to the EP regimen in October 2018. The combination use of bevacizumab was reported to improve the survival data of advanced cervical cancer and was recommended by NCCN guideline. ${ }^{9,15}$ However, after two sessions of the EP chemotherapy, the tumor biomarkers showed increased expression of CEA, CA-125, CA19-9 (Figure 3). The CT (2018-11) (Figure 2B) showed that the treatment outcome was not satisfactory. The CT image showed that part of the lesions including the left supraclavicular lymph nodes, cervix lesions and pelvic lymph node decreased in size while others including bilateral adnexal, implants in the uterine rectal fossa and liver metastases continued to progress.

Considering the poor effect of tumor treatment, we considered that the use of PD-1 inhibitor may be beneficial. After consultation with the patient, we formulated a treatment plan, whereby the chemotherapy regimen of nab-paclitaxel and carboplatin was combined with PD1inhibitor. Pembrolizumab was used as estimated in dose of $2 \mathrm{mg} / \mathrm{kg}$, every 3 weeks following the Chinese clinical trial (KEYNOTE 151). ${ }^{16}$ Nab-paclitaxel and carboplatin were used with proper reduction dose of $260 \mathrm{mg} / \mathrm{m}^{2}$ and $\mathrm{AUC}=5$. On 2018-11-15, 12-10, the patient used pembrolizumab $100 \mathrm{mg} \mathrm{d} 1$, nab-paclitaxel $300 \mathrm{mgd} 2$ and carboplatin 400mgd2. Two cycles after the treatment, the biomarker CA-125 became more than four times lower than before, CEA decreased to almost three times lower than before, and other biomarkers returned to normal value (Figure 3). The gynecological examination suggested that the cervical tumor decreased in size, and the invasion tumor of the vagina was almost completely resolved. The CT scan (Figure 2C) depicted resolved lesions in almost all initial tumor including cervix, the invasion of the vagina, left adnexa, the left supraclavicular lymph nodes, and liver. As the disease got a partial response, this treatment plan was continued for four more sessions, on 20191-7, 2019-1-28, 2019-2-21 and 2019-3-31. The values of the biomarkers gradually decreased, and at the end of the four cycles, only CEA was slightly high (Figure 3). PET/ CT (Figure 2D) obtained before the last session (20193-15) demonstrated almost complete resolution of all lesions. During the treatment, the patient had no obvious side effects, only suffered grade1-2 myelosuppression, with decreased expression of leukocyte and thrombocyte.

As the systematic metastases controlled, pelvic palliative radiotherapy combined with immunotherapy was administered after a multidisciplinary consultation with an aim to control the primary local disease. Chemotherapy was haltered because the patient suffered from grade 1-2 myelosuppression after receiving multiple cycles of chemotherapy. As a consequence, only radiotherapy and immunotherapy were administered to ensure the successful completion of the radiotherapy session. The initial plan of radiotherapy used consisted of whole pelvic field external beam radiotherapy followed with intracavitary brachytherapy. The total dose for external beam radiotherapy was 49.4Gy with a fraction size of $1.9 \mathrm{~Gy}$. As for the brachytherapy, the total dose used was 24 Gy to point A with a fraction size of 6Gy. From 2019-4-11, the patient received Pelvic external beam radiotherapy 1.9Gy daily. After the completion of $43.7 \mathrm{~Gy}$ of external beam radiotherapy, it was noted that the woman suffered grade 3 myelosuppression with thrombocytopenia. Platelets were thus transfused and the planned dose of pelvic external beam radiotherapy was completed. Then, after the patient underwent one brachytherapy of 6Gy, the level of blood platelets dropped to grade4 myelosuppression. At this time, radiotherapy had to be stopped and therefore, the brachytherapy was not completed as planned. The last session of radiotherapy was administered on 2019-05-27. During this period, pembrolizumab was continued to be used Q21days on 4-2, 4-23, 5-16. The myelosuppression persisted and stayed severe, platelet-related drugs were not useful and the patient relied solely on blood transfusion therapy to get a temporary remission. As such, the use of chemotherapy could not be continued. The biomarkers tested on 2019-06-26 showed a rapid increase to an even higher level than what they were before the treatment. Single therapy of pembrolizumab was continued used Q21days on 6-6, 6-23, 7-19 and $8-12$. However, the biomarkers kept on increasing. No obvious 
lesions were observed in the cervix (Figure 5). CT scan showed (Figure 2E) reduced lesion in cervix, the vagina and bilateral adnexa, but with an extensive growth in the liver, supraclavicular lymph nodes, retroperitoneal lymph nodes, and many new lesions were noted in thoracic and peritoneal lymph nodes. Immunotherapy had no effect on the patient anymore and the treatment was interrupted and limited to only maintenance treatment.

\section{Discussion}

Stage IVB cervical cancer is defined as a tumor that metastasizes to distant organs or lymph nodes. ${ }^{17,18}$ These patients are often mixed with recurrent cervical cancer in clinical trials, and therapeutic options are limited. The recommended treatment is based on prior chemotherapy, with cisplatin plus paclitaxel and bevacizumab. ${ }^{19}$ After discussing with a multidisciplinary team, a more specific and adapted treatment can be sought. ${ }^{20}$ However, the prognosis of stage IVB cervical cancer patients remains poor, with 5-year overall survival of about $10 \%{ }^{20}$ The advent of PD1/PD-L1 inhibitors breaks through the therapeutic bottle neck and may change the traditional treatment model. ${ }^{9,21}$

The combination of chemotherapy and immunotherapy has been shown to improve treatment effects. Some studies suggested a synergistic interrelation of chemo and immunotherapy. ${ }^{22,23}$ Especially, in randomized clinical trials, combined PD-L1 blockade with chemotherapy demonstrated significantly increased overall survival and progression-free survival compared to chemotherapy alone with no change in adverse events in non-small cell lung

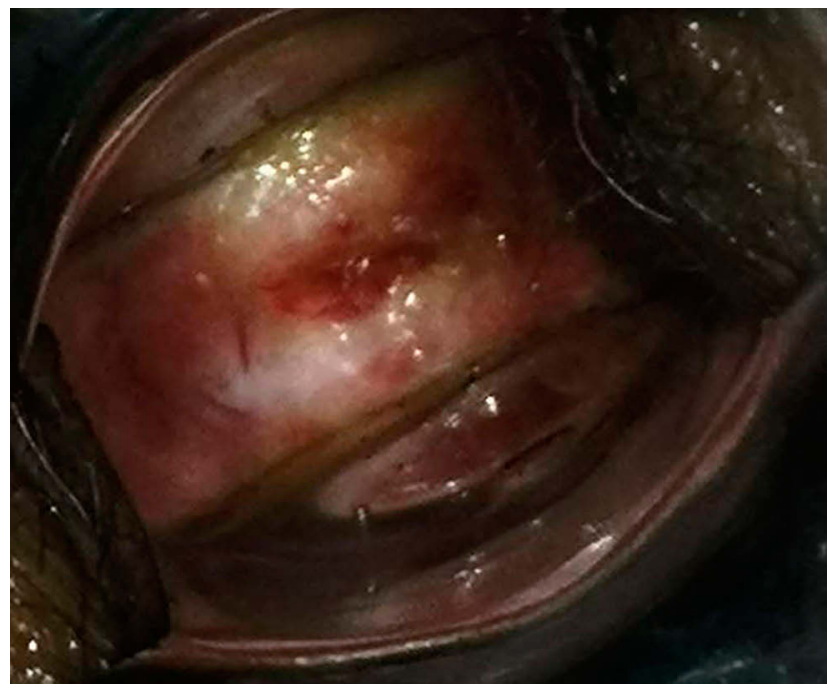

Figure 5 Image of the cervix at the end of therapy. carcinoma. $^{24}$ In addition, preclinical data also suggest a synergistic interaction between radiation therapy and immune checkpoint inhibitors. ${ }^{25,26}$ In stage III non-small cell lung carcinoma, the use of Durvalumab, a PD-L1 inhibitor, following chemo-radiotherapy was found to improve overall survival. ${ }^{25}$

As previously reported, except for some ongoing clinical trials, there are relatively few studies on the application of chemotherapy combined with PD-1/PD-L1 inhibitors in cervical cancer. Here, we presented an innovative case of a patient with cervical cancer stage IVB who was not sensitive to two chemotherapy regimens. Unexpected result happened when the patient turned to use nab-paclitaxel and carboplatin combined with PD-1inhibitor pembrolizumab. After two cycles of treatment, the biomarker decreased significantly, and the imaging report suggested obvious resolution of lesions in almost all initial tumor. After additional four cycles of the regimen, the biomarkers almost returned to normal, and the PET-CT result suggested an almost complete resolution of all lesions. Until the end of the six cycles, the patient had no complaints of any obvious side effects and suffered from only grade1-2 myelosuppression This suggested a positive effect of the combination use of chemotherapy (nab-paclitaxel and carboplatin) and pembrolizumab in this stage IVB cervical cancer patient. Also, this treatment regimen appears to be safe. This is consistent with many clinical trials, as the safety combination use of PD-1/PD-L1 inhibitors with chemotherapy was verified by some articles. $^{24,27}$ Of course, this situation does not rule out the separate effect of albumin paclitaxel. It has been documented that albumin paclitaxel has also proven to be an effective chemotherapy drug for cervical cancer and has been recommended as the second-line therapy for recurrent or metastatic cervical cancer by NCCN guideline. ${ }^{15,28}$

As the systemic metastasis was controlled, local radiotherapy was administered with combination of immunotherapy. Unfortunately, the patient suffered severe grade 4 myelosuppression during this period. Thus, radiotherapy was interrupted and the brachytherapy was not completed. As myelosuppression was not a common adverse event for immunotherapy, ${ }^{29}$ we concluded that radiation therapy triggered off the severe myelosuppression. However, since the patient already received many cycles of chemotherapy which is known to disrupt the platelet-making function, radiotherapy may just aggravate this condition. The combination of radiotherapy and immunotherapy was proved to be effective to this patient as the tumor index indicated a stable condition. However, the safety and proper dose of radiotherapy for patients after cycles of chemotherapy 
and immunotherapy needs to be attentively administered and monitored. Most of the research of radiotherapy and immunotherapy were preclinical trials, the optimal timing of radiation therapy and immune checkpoint inhibitors is unclear. ${ }^{30}$ Many queries remain to be solved such as optimal dose, scheduling and so on.

The tumor index increased significantly after interrupting the use of chemotherapy and radiotherapy. This indicated that the single use of the immune checkpoint inhibitors could not function anymore. The possible reason may be the single effect of immunotherapy was not effective enough. Another possible reason may be the patient developed acquired resistance to immunotherapy. Resistance to immunotherapy is a known phenomenon; however, knowledge is rather limited as to the mechanisms of acquired resistance in patients under treatment. $^{31,32}$

\section{Conclusion}

From this case, we can see a positive effect and good tolerance of the combination use of nab-paclitaxel and carboplatin and pembrolizumab. Radiotherapy with pembrolizumab may also be effective. However, special consideration is needed, as to when be the optimal time for systematic treatment turning to local therapy, how to adjust the dose of chemotherapy and radiotherapy with immunotherapy, and how to avoid acquired resistance of immunotherapy.

\section{Ethics and Consent Statement}

The patient's brother agreed and submitted a written informed consent to allow publication of the details of the case. An institutional approval was not required for a case report.

\section{Acknowledgments}

This study was funded by the National Natural Science Foundation of China (grant number 81702895) and the young talents program of Jiangsu Cancer Hospital.

\section{Disclosure}

The authors report no conflicts of interest in this work.

\section{References}

1. Murfin J, Irvine F, Meechan-Rogers R, Swift A. Education, income and occupation and their influence on the uptake of cervical cancer prevention strategies: a systematic review. J Clin Nurs. 2020;29:393-415.

2. Bray F, Ferlay J, Soerjomataram I, Siegel RL, Torre LA, Jemal A. Global cancer statistics 2018: GLOBOCAN estimates of incidence and mortality worldwide for 36 cancers in 185 countries. CA Cancer J Clin. 2018;68(6):394-424. doi:10.3322/caac.21492
3. Pfaendler KS, Tewari KS. Changing paradigms in the systemic treatment of advanced cervical cancer. Am J Obstet Gynecol. 2016;214 (1):22-30. doi:10.1016/j.ajog.2015.07.022

4. Minion LE, Tewari KS. Cervical cancer - State of the science: from angiogenesis blockade to checkpoint inhibition. Gynecol Oncol. 2018;148(3):609-621. doi:10.1016/j.ygyno.2018.01.009

5. Tewari KS, Sill MW, Long HJ 3rd, et al. Improved survival with bevacizumab in advanced cervical cancer. $N$ Engl J Med. 2014;370 (8):734-743. doi:10.1056/NEJMoa1309748

6. Monk BJ, Sill MW, McMeekin DS, et al. Phase III trial of four cisplatin-containing doublet combinations in stage IVB, recurrent, or persistent cervical carcinoma: a Gynecologic Oncology Group study. J Clin Oncol. 2009;27(28):4649-4655. doi:10.1200/ JCO.2009.21.8909

7. Boussios S, Seraj E, Zarkavelis G, et al. Management of patients with recurrent/advanced cervical cancer beyond first line platinum regimens: where do we stand? A literature review. Crit Rev Oncol Hematol. 2016;108:164-174. doi:10.1016/j.critrevonc.2016.11.006

8. Improved survival with bevacizumab in advanced cervical cancer. N Engl J Med. 2017;377(7):702. doi:10.1056/NEJMx170002

9. Liu Y, Wu L, Tong R, et al. PD-1/PD-L1 inhibitors in cervical cancer. Front Pharmacol. 2019;10:65. doi:10.3389/fphar.2019.00065

10. Okazaki T, Honjo T. PD-1 and PD-1 ligands: from discovery to clinical application. Int Immunol. 2007;19(7):813-824. doi:10.1093/ intimm/dxm057

11. Chung HC, Schellens JHM, Delord J-P, et al. Pembrolizumab treatment of advanced cervical cancer: updated results from the Phase 2 KEYNOTE-158 study. $J$ Clin Oncol. 2018;36(15_suppl):5522. doi:10.1200/JCO.2018.36.15_suppl.5522

12. Frenel JS, Le Tourneau C, O'Neil B, et al. Safety and efficacy of pembrolizumab in advanced, programmed death ligand 1-positive cervical cancer: results from the Phase Ib KEYNOTE-028 trial. $J$ Clin Oncol. 2017;35(36):4035-4041. doi:10.1200/JCO.2017.7 14.5471

13. Hollebecque A, Meyer T, Moore KN, et al. An open-label, multicohort, phase I/II study of nivolumab in patients with virus-associated tumors (CheckMate 358): efficacy and safety in recurrent or metastatic (R/M) cervical, vaginal, and vulvar cancers. J Clin Oncol. 2017;35(15_suppl):5504. doi:10.1200/JCO.2017.35.15_suppl.5504

14. Elsherif S, Odisio EG, Faria S, et al. Imaging and staging of neuroendocrine cervical cancer. Abdom Radiol (NY). 2018;43 (12):3468-3478. doi:10.1007/s00261-018-1667-0

15. Koh WJ, Abu-Rustum NR, Bean S, et al. Cervical cancer, version 3.2019, NCCN clinical practice guidelines in oncology. Natl Compr Canc Netw. 2019;17(1):64-84. doi:10.6004/jnccn.2019.0001

16. Si L, Zhang X, Shu Y, et al. A Phase Ib study of pembrolizumab as second-line therapy for chinese patients with advanced or metastatic melanoma (KEYNOTE-151). Transl Oncol. 2019;12(6):828-835. doi:10.1016/j.tranon.2019.02.007

17. Li H, Wu X, Cheng X. Advances in diagnosis and treatment of metastatic cervical cancer. J Gynecol Oncol. 2016;27(4):e43. doi:10.3802/jgo.2016.27.e43

18. Pecorelli S, Zigliani L, Odicino F. Revised FIGO staging for carcinoma of the cervix. Int J Gynaecol Obstet. 2009;105(2):107-108. doi:10.1016/j.ijgo.2009.02.009

19. Tewari KS, Sill MW, Penson RT, et al. Bevacizumab for advanced cervical cancer: final overall survival and adverse event analysis of a randomised, controlled, open-label, Phase 3 trial (Gynecologic Oncology Group 240). Lancet. 2017;390(10103):1654-1663. doi:10.1016/S0140-6736(17)31607-0

20. Espenel S, Garcia MA, Langrand-Escure J, et al. Special focus on stage IV cervical cancer patients: a decade experience. Oncology. 2019;97(3):125-134. doi:10.1159/000500025

21. Marret G, Borcoman E, Le Tourneau C. Pembrolizumab for the treatment of cervical cancer. Expert Opin Biol Ther. 2019;1-7. 
22. Garassino MC, Torri V, Colombo MP, Sica A. Choosing the best chemotherapy agent to boost immune checkpoint inhibition activity. Cancer Res. 2018;78(20):5729-5730. doi:10.1158/0008-5472.CAN-18-2245

23. Popovic A, Jaffee EM, Zaidi N. Emerging strategies for combination checkpoint modulators in cancer immunotherapy. J Clin Invest. 2018;128(8):3209-3218. doi:10.1172/JCI120775

24. Gandhi L, Rodriguez-Abreu D, Gadgeel S, et al. Pembrolizumab plus chemotherapy in metastatic non-small-cell lung cancer. $N$ Engl $J$ Med. 2018;378(22):2078-2092. doi:10.1056/NEJMoa1801005

25. Antonia SJ, Villegas A, Daniel D, et al. Overall survival with durvalumab after chemoradiotherapy in stage III NSCLC. $N$ Engl J Med. 2018;379(24):2342-2350. doi:10.1056/NEJMoa1809697

26. Kasmann L, Taugner J, Manapov F. Chemo-/immuno-/radiotherapy combination in treatment of solid cancer. Oncotarget. 2019;10 (52):5387-5388. doi:10.18632/oncotarget.27141

27. Pakish JB, Jazaeri AA. Immunotherapy in gynecologic cancers: are we there yet? Curr Treat Options Oncol. 2017;18(10):59. doi:10.1007/s11864-017-0504-y
28. Alberts DS, Blessing JA, Landrum LM, et al. Phase II trial of nab-paclitaxel in the treatment of recurrent or persistent advanced cervix cancer: a gynecologic oncology group study. Gynecol Oncol. 2012;127(3):451-455. doi:10.1016/j.ygyno.2012.09.008

29. Barquin-Garcia A, Molina-Cerrillo J, Garrido P, Garcia-Palos D, Carrato A, Alonso-Gordoa T. New oncologic emergencies: what is there to know about immunotherapy and its potential side effects? Eur J Intern Med. 2019;66:1-8. doi:10.1016/j.ejim.2019.05.020

30. Gunderson AJ, Young KH. Exploring optimal sequencing of radiation and immunotherapy combinations. Adv Radiat Oncol. 2018;3 (4):494-505. doi:10.1016/j.adro.2018.07.005

31. Restifo NP, Smyth MJ, Snyder A. Acquired resistance to immunotherapy and future challenges. Nat Rev Cancer. 2016;16 (2):121-126. doi:10.1038/nrc.2016.2

32. Milano G. Resistance to immunotherapy: clouds in a bright sky. Invest New Drugs. 2017;35(5):649-654. doi:10.1007/s10637-0170456-x

\section{Publish your work in this journal}

OncoTargets and Therapy is an international, peer-reviewed, open access journal focusing on the pathological basis of all cancers, potential targets for therapy and treatment protocols employed to improve the management of cancer patients. The journal also focuses on the impact of management programs and new therapeutic agents and protocols on patient perspectives such as quality of life, adherence and satisfaction. The manuscript management system is completely online and includes a very quick and fair peer-review system, which is all easy to use. Visit http://www.dovepress.com/ testimonials.php to read real quotes from published authors. 